\title{
25 Research Suare \\ NOD2 Genes Mutation in a Blau Syndrome Accompany with Congenital Hyperuricemia: A Case Report
}

\section{Zhaoling Wang}

Zhejiang University School of Medicine Children's Hospital https://orcid.org/0000-0002-6645-1993

MeiPing Lu ( $\sim$ meipinglu@zju.edu.cn)

Zhejiang University School of Medicine Children's Hospital https://orcid.org/0000-0002-4930-9493

\section{Case Report}

Keywords: Blau syndrome, Hyperuricemia, NOD2 gene『case report

Posted Date: January 10th, 2022

DOI: https://doi.org/10.21203/rs.3.rs-1228457/v1

License: (c) (1) This work is licensed under a Creative Commons Attribution 4.0 International License.

Read Full License 


\section{Abstract}

Rationale: Blau syndrome (BS) is a chronic auto-inflammatory granulomatous disorder associated with the nucleotide-binding oligomerization domain-containing 2 (NOD2) gene mutations. Gene mutation is one cause of congenital hyperuricemia, However, the relationship between NOD2 and hyperuricemia was unknown.

Patient concerns: A 3.5-year-old girl was admitted to hospital because of pain in the left calf and red eyes, otherwise, she suffered from skin rash, and skin cysts in the wrist and ankle joints before.

Diagnoses: The girl was diagnosed with sporadic BS (SBS) accompanied with congenital hyperuricemia according to her clinical presentation and gene mutation.

Interventions: The patient received prednisolone combined with adalimumab and methotrexate for controlling SBS. Oral febuxostat to alleviate uric acid levels.

Outcomes: Her serum uric acid decreased to normal levels after 2 weeks with oral febuxostat tablet. Four months following the treatment, the number of cysts was decreased and ocular damage was not progressed.

Lessions: Blau syndrome is a relatively new entity that clinical spectrum continues to expand. This patient provides some information, which would be assist the diagnosis.

\section{Background}

Blau syndrome (BS) is a chronic autosomal dominant auto-inflammatory granulomatous disorder associated with the nucleotide-binding oligomerization domain-containing 2 (NOD2) gene mutations[1]. Hyperuricemia (HUA) in children is associated with age- and sex-related values for uric acid[2]. Herein, we present a case of BS with c.1001G > T(p.R334L) mutation in the NOD2 genes, and this patient also presented with a congenital hyperuricemia, which has not been previously reported.

\section{Case Presentation}

A 3.5-year-old girl was diagnosed with hyperuricemia at her birth. At the age of 6 months, she developed red rash(mild scaly)in face, trunk and extremities and was recorded by a dermatologist. Her skin lesions were treated with topical corticosteroids. At 1 year of age, she developed skin cysts of different sizes in the bilateral wrist and ankle joints. At the age of 3 , she was hospitalized because of pain in the left calf and red eyes.

Rheumatological examination showed numerous brown ichthyosis rash covered the face, trunk and extremities. Subcutaneous nodules were found over the affected joints of wrists and ankles. Ophthalmological examination showed signs of uveitis, keratitis and trichiasis. 
Blood examination revealed a white blood cell count of 11230/ $\mu \mathrm{L}$ (normal: $4000-12000$ ) and C-reactive protein level of $4.21 \mathrm{mg} / \mathrm{L}$ (normal: less than 8). The serum immunoglobulin level was elevated. The immunoglobulin G (lgG) level was elevated to $1620 \mathrm{mg} / \mathrm{dL}$ (normal: 500-1060), lgA level was $150 \mathrm{mg} / \mathrm{dl}$ (normal:34-138), IgM level was $236 \mathrm{mg} / \mathrm{dl}$ (normal:44-144), and IgE level was 101 000mg/dl(normal:010000. The serum uric acid was elevated to $600 \mathrm{umol} / \mathrm{L}$ (normal:155-357), 24 hours of sodium (45mmol/24h, normal: $130-260)$, phosphorus (317.7mg/24h, normal:400-1300), chlorine (53mmol/24h, normal:170-250), and uric acid (865umol/24h, normal:1480-4430) in the urine were decreased. Antinuclear antibody was borderline. Autoantibodies, including anti-dsDNA and anti-cyclic citrullinated peptide, were negative.

Computed tomography (CT) suggested bilateral axillary lymph node enlargement but lung and hilar lymph nodes were negative. Magnetic Resonance Imaging(MRI) showed abnormal signal in bilateral wrist and ankles with bone cysts of right calcaneus bone marrow cavity. B-scan ultrasonography showed vitreous opacity. The whole exome sequencing showed a heterozygous variant in gene NOD2: c.1001G>T(p.R334L). Neither of her parents had the NOD2 mutation.

She was diagnosed with sporadic BS according to her clinical presentation and gene mutaion. Furthermore, we suspected that she has renal excretion dysfunction, but whether it is caused by NOD2 mutation was unknown.

She received the treatment of prednisolone $(0.45 \mathrm{mg} / \mathrm{kg} / \mathrm{d})$ combined with adalimumab (20mg biweekly) and methotrexate( $6.25 \mathrm{mg} /$ week). She was also given febuxostat to lower uric acid levels. The comprehensive therapy was efficacy, the number of cysts was decreased and ocular damage was not progressed. For the first time, her serum uric acid decreased to normal levels after 2 weeks with oral febuxostat tablet.

\section{Discussion}

Blau syndrome(BS) is a chronic auto-inflammatory disorder with autosomal dominant inheritance, first described by Blau in 1985. Sporadic BS (SBS) also called early-onset sarcoidosis (EOS)[3]. It was caused by coding mutation of NOD2 gene, which mapped to chromosomal region 16q12.1-13[1]. The product of NOD2 gene, known as the caspase activation and recruitment domain (CARD15), activates NF-KB after recognizing a signal from a bacterial cell wall component in the leukocyte cytosol[4]. Mutations in the NOD2 gene lead to abnormally enhanced NF-KB activity, which results in the occurrence of BS[5]. This case showed c.1001G > T(p.R334L) mutation in the NOD2 genes. BS is characterized by the clinical triad of recurrent uveitis, dermatitis and arthritis. Other symptoms may be involved, including visceral and vascular involvement[6]. Our patient was born with hyperuricemia, which was not included in the representation of Blau syndrome in the previous report. However, whether congenital hyperuricemia can be caused by it deserves further study.

Congenital hyperuricemia in children and adolescents is associate with chronic disease, including metabolic disease, Down disease, congenital hurt disease and genetic disease[2]. It was already revealed 
that the polymorphisms in the uric acid transporter genes (ABCG2 and SLC2A9) and Apolipoprotein E gene were associated with primary hyperuricemia. In our case, it wasn't found any other mutation except for NOD2 gene[7].

We reported a sporadic form of Blau syndroms in a Chinese girl with missense mutation R334L in the NOD2 gene. In addition to the classical presentation, our patient was born with congenital hyperuricemia which was not previously reported. Blau syndrome is a rare and newly described entity, and its clinical spectrum continues to expand. Our case would be helpful to further better characterize this disease.

\section{Abbreviations}

BS

Blau syndrome

NOD2

nucleotide-binding oligomerization domain-containing 2

SBS

sporadic BS

CT

Computed tomography

MRI

Magnetic Resonance Imaging

EOS

early-onset sarcoidosis

CARD15

caspase activation and recruitment domain

\section{Declarations}

\section{Ethics approval and consent to participate}

The study has obtained approval from the Clinical Research Ethics Committee of Children's Hospital, Zhejiang University School of Medicine (IRB approval no. 2021-IRB-223).

\section{Consent for publication}

the patient and her parents provided written informed consent for the data to be used in analyses and reported.

\section{Availability of data and materials}

All data generated or analyzed during this study are included in this published article. 
The authors have no funding and conflicts of interest to disclose.

\section{Funding}

This work was supported by National Science Foundation of China. (No.82070027)

\section{Authors' contributions}

ZLW was responsible of the collection of clinical information and manuscript preparation. MPL was responsible for critical review of the manuscript.All authors read and approved the final manuscript.

\section{Acknowledgements}

We are grateful for the patient and her family.

\section{References}

1. Poline J, Bourrat E, Meinzer U. Camptodactlyly in Pediatric Practice: Blau Syndrome. J Pediatr. 2020;221:257-9.

2. Kubota M. Hyperuricemia in Children and Adolescents: Present Knowledge and Future Directions. Journal of nutrition metabolism. 2019;2019:3480718.

3. Takeuchi Y, Shigemura T, Kobayashi N, Kaneko N, Iwasaki T, Minami K, Kobayashi K, Masumoto J, Agematsu K: Early diagnosis of early-onset sarcoidosis: a case report with functional analysis and review of the literature. In., vol. 36; 2017: 1189-1196.

4. Takada S, Kambe N, Kawasaki Y, Niwa A, Honda-Ozaki F, Kobayashi K, Osawa M, Nagahashi A, Semi $\mathrm{K}$, Hotta $\mathrm{A}$, et al. Pluripotent stem cell models of Blau syndrome reveal an IFN- $\gamma$-dependent inflammatory. J Allergy Clin Immunol. 2018;141(1):339-49.

5. Kitagawa Y, Kawasaki Y, Yamasaki Y, Kambe N, Takei S, Saito MK: Anti-TNF treatment corrects IFN-Ydependent proinflammatory signatures in Blau. The Journal of allergy and clinical immunology 2021.

6. Poline J, Fogel O, Pajot C, Miceli-Richard C, Rybojad M, Galeotti C, Grouteau E, Hachulla E, Brissaud P, Cantagrel $A$, et al. Early-onset granulomatous arthritis, uveitis and skin rash: characterization of skin. Journal of the European Academy of Dermatology Venereology: JEADV. 2020;34(2):340-8.

7. Toyoda Y, Mančíková A, Krylov V, Morimoto K, Pavelcová K, Bohatá J, Pavelka K, Pavlíková M, Suzuki $\mathrm{H}$, Matsuo $\mathrm{H}$, et al: Functional Characterization of Clinically-Relevant Rare Variants in ABCG2 Identified in a Gout and Hyperuricemia Cohort. CELLS-BASEL 2019, 8(4). 